\title{
Testing Probabilistic Equivalence Through Reinforcement Learning
}

\author{
Josée Desharnais, François Laviolette, \\ and Sami Zhioua \\ IFT-GLO, Université Laval, Québec (QC) Canada, G1K 7P4 \\ \{first_name. last_name\}@ift.ulaval.ca
}

\begin{abstract}
We propose a new approach to verification of probabilistic processes for which the model may not be available. We use a technique from Reinforcement Learning to approximate how far apart two processes are by solving a Markov Decision Process. If two processes are equivalent, the algorithm will return zero, otherwise it will provide a number and a test that witness the non equivalence. We suggest a new family of equivalences, called $K$-moment, for which it is possible to do so. The weakest, 1-moment equivalence, is trace-equivalence. The others are weaker than bisimulation but stronger than trace-equivalence.
\end{abstract}

\section{Introduction}

In program verification, the goal is typically to check automatically whether a system (program, physical device, protocol, etc.) conforms to its pre-established specification. For non-probabilistic systems, one usually expects equivalence between the two, and most of the time this equivalence is chosen to be bisimulation. In the verification of probabilistic systems it has been observed 8 that the comparison between the program and the specification should not be based on equivalences: one reason is that the probabilities involved often come from approximations of the actual numbers. Hence a slight difference in the probabilities between two processes should not necessarily be interpreted as non equivalence. Instead, one is interested in a notion of distance or divergence 11 that quantifies how far apart the processes are. When defining a divergence or distance, we have two focus: its computability of course but also the relation induced by zero distance. The actual value of the distance is usually not relevant but the derived relation, which may be for example bisimulation or trace equivalence, is a guide to evaluate the power or adequacy of the distance or divergence.

In real scenarios, the model of the implementation is rarely known and the available information can only be gathered by interacting with the system. Consequently, verification in this setting has to be based on some form of sampling (or testing). In their famous paper on probabilistic transition systems [13, Larsen and Skou have defined a test language that corresponds to probabilistic bisimulation. Two processes are bisimilar if and only if they accept the same tests

\footnotetext{
${ }^{1}$ We use the word divergence to mean a distance that may not satisfy the triangle inequality and symmetry.
} 
with the same probabilities. From the maximal difference over the probabilities on these tests, Van Breugel et. al. 2] have defined a divergence (in fact a pseudo-metric) that quantifies the difference between the processes. However, the tests defined by Larsen and Skou have a copy construct that represents running many tests on a given state, and this recursively. The need to maintain an arbitrary number of replicas of states is an obstacle to automatisation and has been an argument against bisimulation which is thus considered too strong, even for non-probabilistic processes.

In this paper, we explore alternative equivalences and divergences that do not require to maintain an unbounded number of replicas. In particular, we suggest a new family of equivalences called $K$-moment that only need a nonrecursive form of replication. We also propose an algorithm to compute these divergences using Reinforcement Learning (RL) methods; these are applicable even when the model is not available. While verification techniques can deal with processes of about $10^{12}$ states, RL algorithms do a lot better; for example, the TD-Gammon program deals with more than $10^{40}$ possible states [15]. The key idea of our approach is to define a Markov Decision Process (MDP) out of the processes to be tested and to interpret the optimal value of this MDP as a divergence between the processes. Moreover, the algorithm we propose outputs a test that witnesses the computed divergence. In [5, we showed how it can be done for trace-equivalence; we now extend the approach to a large family of testing equivalences.

The plan of the paper is as follows. In the following section, we point out the difficulties behind testing probabilistic bisimulation and define the $K$-moment equivalence. In Section 3, we informally expose our approach via a one player stochatic game and give the key definition of the MDP, the associated theorems, and the experimental results. Section 4 shows briefly how the approach can be applied to other equivalence notions.

\section{Testing Equivalences}

We consider probabilistic reactive systems where actions are meant to be synchronized through interaction with the environment and where no internal actions occur. Our models are Labelled Markov Processes (LMPs) [1] while they can be uncountable in general, we restrict ourselves to countable ones. Finite LMPs are also called Probabilistic labelled transition systems or Markov decision processes without rewards. A countable LMP is a tuple $(S, \mathrm{i}$, Act, $P)$ where $S$ is a countable set of processes, $\mathrm{i} \in S$ the initial process, Act a finite set of actions, and $P(s, a)$ a sub-probability distribution on $S$, for $s \in S$ and $a \in A c t$ (see Fig. 11). We will write $\mathcal{S}$ generically for this tuple, and assume that subscripts propagate to the four elements. We use the notation $P_{s X}(a)$ for $P(s, a)(X)$, the probability that an $a$-transition from $s$ ends in $X$. Given a trace $\tau$ (i.e., a sequence of actions), $P_{\text {is }}(\tau)$ is the probability to reach $s$ with $\tau$ from the initial state i. We will always assume our models to be tree like; up to bisimulation [1], it is always possible. 


\section{Bisimulation}

Larsen and Skou showed that probabilistic bisimulation can be characterized by a testing scenario [13]. Their test language has the following syntax:

$$
\mathcal{T}_{L S}: \quad t::=\omega \mid \text { a.t } \mid\left(t_{1}, \ldots, t_{n}\right)
$$

$\omega$ is a dummy test that always terminates with success; test a.t consists in executing action $a$ and, in case of success, proceeding with test $t$; finally, test $\left(t_{1}, \ldots, t_{n}\right)$ consists in making $n$ copies of the current process and then executing test $t_{i}$ on the $i^{t h}$ copy for $i=1, \ldots, n$. The execution of a test may result in several possible observations. Let $a^{\checkmark}$ represents the success of action $a$ and $a^{\times}$ its failure. The observation set of test $t$ is recursively defined as follows :

$O_{\omega}=\{\omega\}, \quad O_{a . t}=\left\{a^{\times}\right\} \cup\left\{a^{\checkmark} . e \mid e \in O_{t}\right\}, \quad O_{\left(t_{1}, \ldots, t_{n}\right)}=O_{t_{1}} \times \ldots \times O_{t_{n}}$

To each test $t$ is associated a probability distribution $Q_{t}^{s}(e)$ on $O_{t}$; it represents the probability to witness observation $e$ after running $t$ on process $s$ and is defined as :

$$
\begin{gathered}
Q_{\omega}^{s}(\omega)=1, \quad Q_{\left(t_{1}, \ldots, t_{n}\right)}^{s}\left(\left(e_{1}, \ldots, e_{n}\right)\right)=\prod_{i} Q_{t_{i}}^{s}\left(e_{i}\right) \text { where } e_{i} \in O_{t_{i}} \forall i \\
Q_{a . t}^{s}\left(a^{\times}\right)=1-P_{s S}(a), \quad Q_{a . t}^{s}\left(a^{\checkmark} . e\right)=\sum_{s^{\prime} \in S} P_{s s^{\prime}}(a) Q_{t}^{s^{\prime}}(e) \text { where } e \in O_{t} .
\end{gathered}
$$

Theorem 1 ([13]). Two processes are probabilistic bisimilar iff they yield the same probability distribution on observations for any test of the grammar $\mathcal{T}_{L S}$.

The replication construct in the test language $\mathcal{T}_{L S}$ makes bisimulation very difficult to check in practice. Indeed, this construct requires to make $n$ replicas of the current process and to execute a test on each replica. Since this construct is defined recursively on tests, there is no bound on the number of replicas that must be kept in memory. However, as is well known, bisimulation cannot be tested without recursive replication. In this work, we propose equivalences that make sure to limit the number of replicas 2 . This allows us to propose an algorithm that uses efficient techniques of RL to estimate the divergence between LMPs. A major advantage of this approach is that it runs even if the model of the implementation is unknown, contrarily to Van Breugel and Worrell's work [3]. In the latter, a pseudo metric that corresponds to bisimulation is computed through a linear programming algorithm.

\section{$\boldsymbol{K}$-moment Equivalences}

Trace-equivalence, contrarily to bisimulation, can be tested without the need to create replicas recursively, but for many applications, it does not discriminate enough. Especially, it cannot discriminate between the following two processes:

\footnotetext{
${ }^{2}$ In the classification of Van Glabbeek 9, the testing machine we assume is equipped with (1) a series of buttons (one for each action), (2) a reset button and (3) a replication button to generate copies of the current process. To avoid recursive replication, we delete copies in memory once a transition happens from one state to the next.
} 

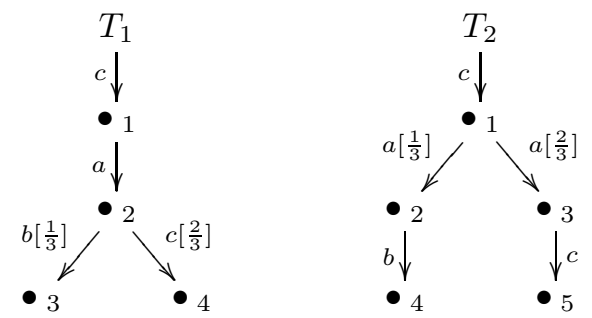

Of course bisimulation does discriminate between them, and therefore can catch the fact that the probabilistic choice happens at different levels in both processes. One may wonder if equivalences without recursive replication can catch this difference. Such equivalences, if they can be tested efficiently, should be a good compromise between the fact that bisimulation is very costly to test and the fact that trace-equivalence does not discriminate enough.

It is well known that probabilistic bisimulation can be formulated as the greatest fixed point of $F$ defined as follows. Given an equivalence relation $R$, we say that two states $s_{1}, s_{2}$ are $F(R)$ equivalent if they have the same probability to jump to an equivalence class of $R$ with every sequence of actions. More formally $\sum_{t \in C} P_{s_{1} t}(\tau)=\sum_{t \in C} P_{s_{2} t}(\tau)$ for all $R$-equivalence class $C$. Observe that traceequivalence is simply $F(S \times S)$. Hence, in order to naturally define an equivalence whose discrimination power is between bisimulation and trace-equivalence, one can consider $\cap_{a \in A c t} F\left(\sim_{a}\right)$ where $\sim_{a}$ is the equivalence that identifies states that have the same total probability to perform action $a$, that is $P_{s S}(a)$. This relation does discriminate $T_{1}$ and $T_{2}$ and any two trace-equivalent processes on which probabilistic choices happen at different levels. It can be tested without recursive replicas but, unfortunately, the number of needed replicas at a particular state is unbounded. However, we will see that it is the limit of a sequence of "bounded replicas" equivalences. First we need the following:

Definition 1. Let $(S, \mathrm{i}, A c t, P)$ be an $L M P, a \in A c t$ and $\tau \in A c t^{*}$. We define $X_{\tau, a}: S \cup\{$ Dead $\} \rightarrow[0,1]$ the random variable representing the probability to perform action a after having run trace $\tau$. Dead is the outcome of the experiment of failing to perform $\tau$. Equivalently, for $p>0$

$$
\operatorname{Pr}\left(X_{\tau, a}=p\right)=\sum_{s: P_{s S}(a)=p} P_{i s}(\tau)
$$

and $\left\{X_{\tau, a}=0\right\}=\left\{s: P_{s S}(a)=0\right\} \cup\{$ Dead $\}$.

At first sight, it is surprising to see a random variable taking probability values, but recall that we are performing tests on processes and are indeed observing the probabilities that these tests be accepted.

It is easy to see that two processes $\mathcal{S}_{1}$ and $\mathcal{S}_{2}$ are $\cap_{a \in A c t} F\left(\sim_{a}\right)$-equivalent if, and only if, for any trace $\tau \in A c t^{*}$ and action $a \in A c t$, the random variables $X_{\tau, a}^{\mathcal{S}_{1}}$ and $X_{\tau, a}^{\mathcal{S}_{2}}$ are equal. Since any two discrete random variables are equal if and only if they have the same moment: 3 , the following is a natural relaxation of the preceding equivalence:

\footnotetext{
${ }^{3}$ Recall that, the $i^{\text {th }}$ moment of a random variable $X$ is the expected value of $X^{i}$.
} 
Definition 2. Let $K \in \mathbb{N}^{*}$. Two LMPs $\mathcal{S}$ and $\mathcal{S}^{\prime}$ are $K$-moment equivalent, if and only if, $\forall \tau \in A c t^{*}, \forall a \in A c t \quad X_{\tau, a}^{\mathcal{S}}$ and $X_{\tau, a}^{\mathcal{S}^{\prime}}$ have exactly the same first $K$ moments. That is, $E\left(\left(X_{\tau, a}^{s}\right)^{k}\right)=E\left(\left(X_{\tau, a}^{s^{\prime}}\right)^{k}\right)$ for $k \leq K$, or equivalently, $\sum_{s \in S} P_{\mathrm{i} s}(\tau)\left(P_{s S}(a)\right)^{k}=\sum_{s^{\prime} \in S^{\prime}} P_{\mathrm{i}^{\prime} s^{\prime}}^{\prime}(\tau)\left(P_{s^{\prime} S^{\prime}}^{\prime}(a)\right)^{k}$ for $k \leq K$.

It is easy to see that 1-moment equivalence corresponds to trace-equivalence. $K$-moment also has a nice characterization in term of tests:

Definition 3. Let $K \in \mathbb{N}^{*}$. The $K$-moment test grammar is defined as:

$$
\mathcal{T}_{\text {Kmoment }}: \quad t::=\omega \mid a^{k} . t
$$

with $k \leq K$ such that (1) the test $a^{k} . t$ consists in running action a on $k$ copies of the current process and if the last action succeeds, proceed with test $t$ on the last copy (and delete the other copies);

(2) the observations corresponding to the test $a^{k} . t$ are given by:

$$
O_{a^{k} . t}=\left\{a^{k^{\times}}\right\} \cup\left\{a^{k^{\star}} . e \mid e \in O_{t}\right\} \cup\left\{a^{k^{\curlyvee}} . e \mid e \in O_{t}\right\}
$$

$a^{k^{\times}}$is the observation that the last action has failed (and maybe others); $a^{k^{\star}}$ means that an action failed but not the last one and finally $a^{k^{r}}$ is the observation that all copies succeeded on a. The probability distribution on observations is:

- $Q_{a^{k} \cdot t^{s}}\left(a^{k^{\times}}\right)=1-P_{s S}(a)$

- $Q_{a^{k} . t}^{s}\left(a^{k^{\star}} . e\right)=\left(1-P_{s S}(a)^{k-1}\right) \sum_{s^{\prime} \in S} P_{s s^{\prime}}(a) Q_{t}^{s^{\prime}}(e)$

- $Q_{a^{k} . t}^{s}\left(a^{k^{r}} . e\right)=P_{s S}(a)^{k-1} \sum_{s^{\prime} \in S} P_{s s^{\prime}}(a) Q_{t}^{s^{\prime}}(e)$.

For processes $T_{1}$ and $T_{2}$ defined above, the test $t=c \cdot a \cdot b^{2} . \omega$ yields different probability distributions on observations: $Q_{t}^{T_{1}}\left(c^{\checkmark} \cdot a^{\checkmark} \cdot b^{2^{\vee}} \cdot \omega\right)=11\left(\frac{1}{3}\right)^{2} 1=\frac{1}{9}$ while $Q_{t}^{T_{2}}\left(c^{\checkmark} \cdot a^{\checkmark} \cdot b^{2^{\checkmark}} \cdot \omega\right)=1 \frac{1}{3}(1)^{2} 1=\frac{1}{3}$. Note also that $Q_{t}^{T_{1}}\left(c^{\checkmark} \cdot a^{\checkmark} \cdot b^{2^{\vee}} \cdot \omega\right)=$ $11\left(\frac{2}{3} \frac{1}{3}\right) 1$, whereas $Q_{t}^{T_{1}}\left(c^{\checkmark} \cdot a^{\checkmark} \cdot b^{2^{\times}}\right)=11\left(1-\frac{1}{3}\right)$.

Theorem 2. Let $K \in \mathbb{N}^{*}$. Two LMPs are $K$-moment equivalent iff they yield the same probability distribution on observations of tests generated from $\mathcal{T}_{\text {Kmoment }}$.

This equivalence is interesting in two ways: it closes the gap between bisimulation and trace-equivalence and it is testable without recursive replication. We will now take advantage of its testability.

\section{Testing Without the Model}

We want to define a divergence between "Spec", a model of the specification and "Impl", a real system; the model of the latter is not necessarily available but we can interact with it (as a black-box) via the testing machine. We also want this divergence to come as the solution of an MDP, the basic ingredient of RL techniques, on which the learning algorithm works. The MDP will be defined in Section 3.2. The rewards in the MDP have to be chosen carefully to make sure that the optimal value will indeed define a divergence as we expect: this is the subject of Section 3.1. We expose our approach in the form a one-player stochastic game, the player being the personification of the learning algorithm. 
We illustrate our approach for $K$-moment equivalence but the ideas can be adapted for other equivalences testable through a recursive replication-free test grammar (see Section 4).

\subsection{K-Moment Equivalences Through a Stochastic Game}

When interacting with processes "Spec" and "Impl", the player's goal is to detect difference between the two. Hence the game (and its corresponding MDP) should give him low reward when they behave the same (i.e., both succeed or both fail) and high reward otherwise. However, the processes are probabilistic and hence a process may behave differently on different trials of the same action, which could lead the player to find a big difference between identical processes. This will happen more likely when the choice at a state is "wide", more technically, when the entropy is high. To compensate this uncertainty, we introduce a third process, called "Clone" which is simply a copy of the specification but given in the form of a black-box (exactly as "Impl") (see Figure 11). The player will get a high reward if "Impl" and "Spec" differ for some action, but this reward could be cancelled if "Spec" and "Clone" also do. Recall that the player does not see the states reached in "Impl" and "Clone" but does see what happens in "Spec".

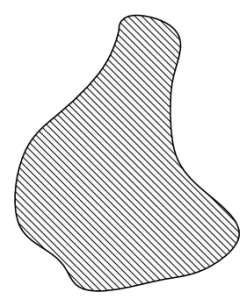

Impl

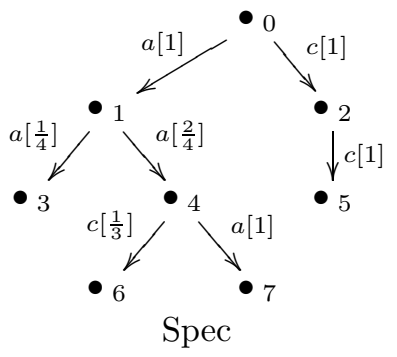

Spec

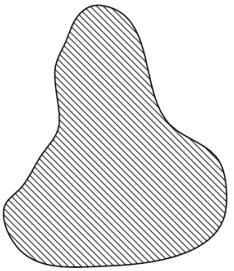

Clone

Fig. 1. Implementation, Specification, and Clone

Game $_{\text {Kmoment }}$ : The three processes start in their initial states; then

Step 1: The player chooses an action $a$ and an integer $k<K$, and makes a prediction Pred on its success or failure on "Spec" . We will denote the player choice by $a^{k^{\gamma}}$ for success and by $a^{k^{\times}}$otherwise.

Step 2: $a^{k}$ is run on "Impl", "Spec" and "Clone" as in Definition [3 Let $\left(o_{I}, o_{S p}, o_{C}\right) \in\left\{a^{k^{\star}}, a^{k^{\star}}, a^{k^{\curlyvee}}\right\}^{3}$ be the outcome of this experiment.

Step 3: Get reward as defined below. If the last occurrence of action $a$ succeeds on the three processes, go to Step 1 with the three processes in their reached state. Else the game ends.

The player gets a reward according to the following formula:

$$
\left.R:=\left(o_{S p} \sim \text { Pred }\right)\left(\left(o_{I} \nsim o_{S p}\right)\right)-\left(o_{S p} \nsim o_{C}\right)\right)
$$

where 0 and 1 are used as both truth values and numbers and the relation $\sim$ is a relaxation of equality to identify cases where a failure happens: i.e., $a^{k^{\times}} \sim a^{k^{\star}} \nsim a^{k^{\curlyvee}}$. 
For example, if $a^{k^{\natural}}$ is selected and the observation is $\left(a^{k^{\downarrow}}, a^{k^{\natural}}, a^{k^{\curlyvee}}\right)$ we obtain a reward of $\left(a^{k^{\gamma}} \sim a^{k^{\gamma}}\right)\left(\left(a^{k^{\downarrow}} \nsim a^{k^{\gamma}}\right)-\left(a^{k^{\gamma}} \nsim a^{k^{\gamma}}\right)\right)=1(1-0)=1$, but for $\left(a^{k^{\star}}, a^{k^{\times}}, a^{k^{\curlyvee}}\right)$, we obtain $0(0-1)=0$.

With the rewards so defined, we will show in Section 3.2 that "Spec" and "Impl" are $K$-moment equivalent if, and only if, the optimal strategy has expected reward zero. In other words, we will show that the optimal value of the MDP defined from this game yields a suitable notion of divergence.

Remark 1. It is not clear at first sight why the prediction is important in Step 1. It happens that by just running $a^{k}$ on the processes and collecting the rewards we do not reach our goal totally (that is, without multiplying by $o_{S p} \sim$ Pred). If "Spec" and "Impl" are $K$-moment equivalent, the optimal strategy would indeed have expected reward zero, as wanted. However, the converse would not be true: there are $K$-moment inequivalent LMPs for which the optimal strategy would have expected reward zero. This is not what we want because they would get a divergence zero. Here is an example: for $K=1$, consider three systems with one $a$-transition from one state to another one. The probability of this transition is $\frac{1}{2}$ in "Spec" (and "Clone") and 1 in "Impl". Note that such examples all exhibit a specific form of symmetry in "Spec".

Remark 2. This game has been inspired by a well known and well studied divergence, the Kullback-Leibler divergence. The idea is that two processes are "equivalent" via testing if, and only if, they yield the same probability distributions on observations for any test generated from the given test grammar. Hence, the divergence between two processes could be defined with the help of a divergence between the probability distributions on test observations. The Kullback-Leibler divergence (KL divergence) would be a candidate: it is defined, for two distributions $Q$ and $P$, as $\mathrm{KL}(Q \| P):=\mathrm{E}_{h \sim Q} \ln \frac{1}{P(h)}-\mathrm{E}_{h \sim Q} \ln \frac{1}{Q(h)}$ [4. Unfortunately, because of the high number of possible tests (on huge systems), the maximum value over all Kullback-Leibler divergences is not tractable. Nevertheless, let us describe the analogy between $\mathbf{G a m e}_{\text {Kmoment }}$ and the KL divergence. The entropy of $P$ relativised by $Q\left(\mathrm{E}_{h \sim Q} \ln \frac{1}{P(h)}\right.$ in the above formula) can be seen as how likely we can obtain different observations when interacting (via some test $t$ ) with both "Spec" and "Impl". On the other hand, the entropy of the distribution $Q\left(\mathrm{E}_{h \sim Q} \ln \frac{1}{Q(h)}\right.$ in the above formula) can also be seen as a quantification over the likelihood to obtain different observations when running the same action on "Spec" twice. Thus, the game expresses the same kind of tradeoff as the KL divergence. This is a first indication that one can derive the notion of divergence we are looking for.

\subsection{The Reinforcement Learning Framework}

In artificial intelligence, Markov Decision Processes (MDPs) offer a popular mathematical tool for planning and learning in the presence of uncertainty [11. MDPs are a standard formalism for describing multi-stage decision making in probabilistic environments (what we called a one-player stochastic games in the 
preceding section). The objective of the decision making is to maximize a cumulative measure of long-term performance, called the reward.

In an MDP, an agent interacts with a stochastic environment at a discrete, low-level time scale. On each time step $t$, the agent observes its current state $s_{t} \in S$ and chooses an action $a_{t}$ from an action set $A$. One time step later, the agent transits to a new state $s_{t+1}$, and receives a reward $r_{t+1}$. For a given state $s$ and action $a$, the expected value of the immediate reward is denoted by $R_{s S}^{a}$ and the transition to a new state $s^{\prime}$ has probability $\operatorname{Pr}_{s s^{\prime}}^{\mathcal{M}_{\mathcal{K}}}(a)$, regardless of the path taken by the agent before state $s$ (this is the Markov property). The goal in solving MDPs is to find a way of behaving, or policy, which yields a maximal reward. Formally, a policy is defined as a probability distribution for picking actions in each state. For any policy $\pi: S \times A \rightarrow[0,1]$ and any state $s \in S$, the value function of $\pi$ for state $s$ is defined as the expected infinite-horizon discounted return from $s$, given that the agent behaves according to $\pi: V^{\pi}(s):=E_{\pi}\left\{r_{t+1}+\gamma r_{t+2}+\gamma^{2} r_{t+3}+\cdots \mid s_{t}=s\right\}$ where $i$ is the initial state and $\gamma$ is a factor between 0 and 1 used to discount future rewards. The objective is to find an optimal policy, $\pi^{*}$ which maximizes the value $V^{\pi}(s)$ of each state $s$. The optimal value function, $V^{*}$, is the unique value function corresponding to any optimal policy.

If the MDP has finite state and action spaces, and if a model of the environment is known (i.e., state space $S$, immediate rewards $R_{s s^{\prime}}^{a}$ and transition probabilities $\operatorname{Pr}_{s s^{\prime}}^{\mathcal{M}_{\mathcal{K}}}(a)$ ), then DP algorithms (namely policy evaluation [15]) can compute $V^{\pi}$ for any policy $\pi$. Similar algorithms can be used to compute $V^{*}$. RL methods, in contrast, compute approximations to $V^{\pi}$ and $V^{*}$ directly based on the interaction with the environment, without requiring a complete model or finiteness of the MDP. Only the state space of the MDP and the knowledge of the exact current state at each step of the interaction are required. This is exactly what we are looking for.

Constructing the MDP. We now define the MDP (denoted $\mathcal{M}_{K}$ ) with which the divergence between two LMPs, "Impl" and "Spec", will be computed. In-

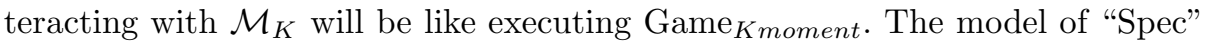
is needed and must be in a tree-like representation (up to bisimulation, this is always possible). Since it has a tree-like structure, for any of its state $s$, there is a unique sequence of actions (or trace, denoted tr.s) from the initial state to $s$. For the LMP "Impl", only the knowledge of all possible conditional probabilities $P^{I}\left(a^{k^{\gamma}} \mid \tau\right)$ and $P^{I}\left(a^{k^{\times}} \mid \tau\right)$ of observing the success or failure of an action $a^{k}$ given any successfully executed trace $\tau$ is required; note that we define the trace corresponding to the execution of the $i$ first steps of the test $a_{1}^{k_{1}^{-}} a_{2}^{k_{2}} \ldots a_{n}^{k_{n}^{-}}$as $\tau=a_{1} a_{2} \ldots a_{i}$ (we write $a^{k^{-}}$for $a^{k^{\natural}}$ or $\left.a^{k^{k^{x}}}\right)$. We write $P^{C}\left(a^{k^{k}} \mid \tau\right)$ and $P^{C}\left(a^{k^{x}} \mid \tau\right)$ for the same conditional probabilities but on a copy of the first LMP (called "Clone"): this is for readability and is no additional information since $P^{C}\left(a^{\checkmark} \mid \tau\right)=P^{S p e c}\left(a^{\checkmark} \mid \tau\right)$.

The state space of the MDP $\mathcal{M}_{K}$ will be the state space of the LMP "Spec" plus one extra state, called Dead. This state corresponds in the MDP to the fact that Game Kmoment $_{\text {is }}$ over: i.e., given that $a^{k^{k}}$ is the last choice of the player, then one of the three LMPs failed to execute the $k^{t h}$ action $a$. Any other state 
of $\mathcal{M}_{K}$ represents the current state of the LMP "Spec" during the execution of Game $_{\text {Kmoment }}$. The probability transitions $\left(\operatorname{Pr}_{s s^{\prime}}^{\mathcal{M}_{\mathcal{K}}}\left(a^{k^{-}}\right)\right)$and the average rewards signals $\left(R_{s S}^{a^{k^{-}}}\right)$therefore follows from the rules of Game Kmomen $_{4}$. More formally:

Definition 4. Given "Impl", "Spec" = (States, i, Actions, Pr Spec $)$, and "Clone", the set of states of the MDP $\mathcal{M}_{K}$ is $S:=$ States $\cup\{$ Dead $\}$, with initial state i; the action set is Act $:=\left\{a^{k^{\vee}} \mid a \in\right.$ Actions, $\left.1 \leq k \leq K\right\} \cup\left\{a^{k^{\times}} \mid a \in\right.$ Actions, $1 \leq$ $k \leq K\}$. The next-state probability distribution is the same for $a^{k^{\vee}}$ and $a^{k^{\times}}$; it is defined below, followed by the definition of the reward function.

$$
\begin{aligned}
& \operatorname{Pr}_{s s^{\prime}}^{\mathcal{M}_{\mathcal{K}}}\left(a^{k^{-}}\right):= \begin{cases}\operatorname{Pr}_{s s^{\prime}}^{\text {Spec }}(a) P^{I}\left(a^{\checkmark} \mid \text { tr.s }\right) P^{C}\left(a^{\checkmark} \mid \text { tr.s }\right) & \text { if } s^{\prime} \neq \text { Dead } \\
1-P^{S p e c}\left(a^{\checkmark} \mid s\right) P^{I}\left(a^{\checkmark} \mid \text { tr.s }\right) P^{C}\left(a^{\checkmark} \mid \text { tr.s }\right) & \text { if } s^{\prime}=\text { Dead }\end{cases} \\
& R_{s s^{\prime}}^{a^{k^{-}}}:= \begin{cases}P^{\operatorname{Spec}}\left(a^{(k-1)^{-}} \mid s\right) \Delta_{t r . s}^{a^{(k-1)^{-}}} & \text {if } s^{\prime} \neq \text { Dead and } k>1 \\
0 & \text { if } s^{\prime} \neq \text { Dead and } k=1\end{cases} \\
& R_{s \text { Dead }}^{a^{k^{-}}}:=\frac{1}{\operatorname{Pr}_{\text {sDead }}^{\mathcal{M} \mathcal{K}}\left(a^{k^{-}}\right)}\left(P^{S p e c}\left(a^{k^{-}} \mid s\right) \Delta_{\tau}^{a^{k^{-}}}-\sum_{s^{\prime} \in S \backslash\{\text { Dead }\}} \operatorname{Pr}_{s s^{\prime}}^{\mathcal{M}_{\mathcal{K}}}\left(a^{k^{-}}\right) R_{s s^{\prime}}^{a^{k^{-}}}\right)
\end{aligned}
$$

where $\bullet P^{S p e c}\left(a^{k^{\curlyvee}} \mid s\right):=\sum_{s^{\prime} \in S \backslash\{\text { Dead }\}} \operatorname{Pr}_{s s^{\prime}}^{\text {Spec }}(a)$, and $P^{S p e c}\left(a^{k^{\times}} \mid s\right):=1-P^{S p e c}\left(a^{k^{\curlyvee}} \mid s\right)$, on a state $s$,

$$
\text { - } \Delta_{\tau}^{a^{k^{\curlyvee}}}:=P^{C}\left(a^{k^{\curlyvee}} \mid \tau\right)-P^{I}\left(a^{k^{\curlyvee}} \mid \tau\right) \text { and } \Delta_{\tau}^{a^{k^{`}}}:=-\Delta_{\tau}^{a^{k^{\triangleleft}}} \text {. }
$$

The proof that the formal definition of $\mathcal{M}_{K}$ corresponds to the intuition given before Definition 4 is omitted as well of the proof of the following theorem 5 .

Theorem 3. Let $\mathcal{M}_{K}$ be the MDP induced by "Spec", "Impl", and "Clone". If $\gamma<1$ or $\left|\mathcal{M}_{K}\right|<\infty$ then $V^{\star}(\mathrm{i}) \geq 0$ for any policy $\pi$, and $V^{\star}(\mathrm{i})=0$ if and only if "Spec" and "Impl" are K-moment equivalent.

We can now give the definition of the central notion of this paper.

Definition 5. Let "Spec" and "Impl" be two LMPs and $\mathcal{M}_{K}$ their induced MDP. We define their $K$-moment equivalence divergence as

$$
\operatorname{div}_{K \text {-moment }}\left(\text { "Spec" } \mid \text { "Impl") }:=V^{\star}(\mathrm{i})\right. \text {. }
$$

\subsection{The Choice of the Learning Algorithm and PAC Guaranties}

As mentioned in Section 3.1, the full model of the MDP might not be available. Therefore, it is not appropriate to use a Dynamic Programming algorithm such as value iteration [15] to solve the MDP. Instead, we use a Q-Learning algorithm [16. Q-Learning is an off-policy Temporal Difference (TD) control algorithm which directly approximates $V^{\star}(\mathrm{i})$. The algorithm has been proven to converge

\footnotetext{
${ }^{4}$ If one only wants to run a Q-learning algorithm on it, only the model of "Spec" and a possibility of interacting with "Clone" and "Impl" is required.

${ }^{5}$ See http://www.ift.ulaval.ca/ laviolette/Publications/publications.html.
} 
to the optimal value [17. Moreover, some results about its convergence rates have been proposed [6]. However, in the field of verification, the main goals are $(*)$ to find the difference between the implementation of a system and its specification and also $(* *)$ to have a guarantee on the fact that this difference is very small in the case where we do not find any such difference during the investigation. Hence, from that perspective, a PAC-guarantee is the most appropriate tool.

Definition 6. We say that we have a PAC (Probably Approximately Correct) guarantee for a learning algorithm on an MDP $\mathcal{M}$ if, given an a priori precision $\epsilon>0$ and a maximal probability error $\delta$, there exists a function $f\left(\mathcal{M}_{K}, \epsilon, \delta\right)$ such that if the number of episodes is greater than $f\left(\mathcal{M}_{K}, \epsilon, \delta\right)$, then

$$
\operatorname{Prob}\left\{\left|\overline{V^{\hat{\pi}}(\mathrm{i})}-V^{\star}(\mathrm{i})\right| \leq \epsilon\right\} \geq 1-\delta
$$

where $\hat{\pi}$ is the policy returned by the Q-learning algorithm and $\overline{V^{\hat{\pi}}(\mathrm{i})}$ is the estimation of $V^{\star}(\mathrm{i})$ given by this algorithm.

The Q-learning algorithm does have a PAC guarantee [12, but the function $f\left(\mathcal{M}_{K}, \epsilon, \delta\right)$ is very difficult to compute, which makes this guarantee unusable in practice. The Fiechter RL algorithm [7] comes with a simpler PAC guarantee and hence one can use it in the current setting. The main drawback of the Fiechter algorithm remains its inefficiency compared to Q-Learning.

However we can still reach goal $(*)$ using any RL learning algorithm. Indeed, when the processes are not $K$-moment equivalent, we can guarantee a bottom bound for the optimal value using Hoeffding inequality based on the following idea. Let $\hat{\pi}$ be the policy returned by the RL algorithm. Let $\overline{V^{\hat{\pi}}(\mathrm{i})}$ be the estimation of $V^{\hat{\pi}}(\mathrm{i})$ using a Monte Carlo [15] algorithm with $m$ episodes. Given $\epsilon, \delta \in] 0,1\left[\right.$, according to the Hoeffding inequality, if $m \geq \frac{1}{\epsilon^{2}} \ln \left(\frac{2}{\delta}\right)$, we have Equation (11) with $V^{\star}(\mathrm{i})$ replaced with $V^{\hat{\pi}}(\mathrm{i})$. Since $V^{\hat{\pi}}(\mathrm{i})$ never exceeds the optimal value $V^{\star}(\mathrm{i})$, we have the PAC guarantee: $\operatorname{Prob}\left\{\overline{V^{\hat{\pi}}(\mathrm{i})}-V^{\star}(\mathrm{i}) \leq \epsilon\right\} \geq 1-\delta$.

Experimental Results. The approach described so far has been implemented for the trace and $K$-moment family equivalences. Two action selection algorithms have been experimented: $\epsilon$-greedy and SoftMax. For both methods, we tried several functions to decrease the $\epsilon$ (resp. the $\tau$ ) values. The combination that produced the best results is SoftMax such that the temperature $\tau$ is decreasing from 0.8 to 0.01 according to the function : $\tau=\frac{k}{\text { currentEpisod }+l}$ ( $k$ and $l$ are constants). The learning rate $\alpha$ (also called step size) must decrease in order to assure convergence of the Q-Learning algorithm. We tried several decreasing functions and the best convergence results are with $\frac{1}{x}$ where $x$ is the number of times the state-action has been visited. The two following graphics

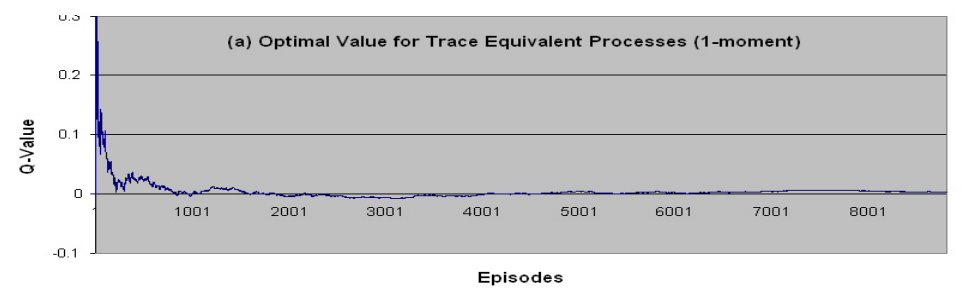




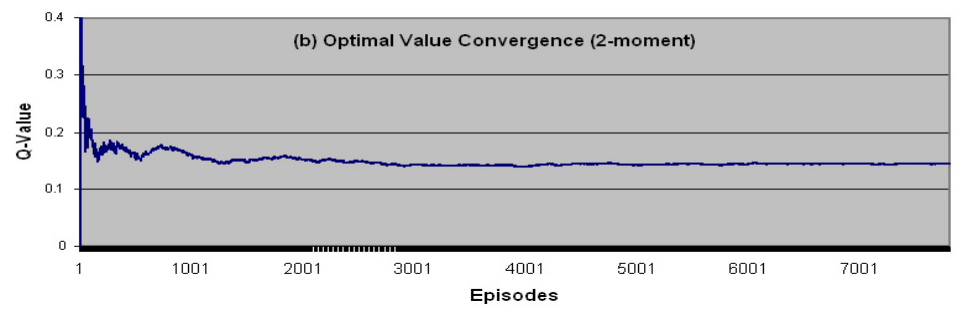

show how the Q-Learning algorithm converges to the optimal value. In the above graphics, we tracked the optimal value in one execution of 10000 episodes on small examples. Running the algorithm with 1-moment option on trace equivalent processes produces the graphic (a). It is easy to see that the estimated divergence value converges to zero as expected. The second graph (b) is obtained by running the algorithm with 2-moment option and in this case, however, the estimated value converges to a value bigger than zero indicating a difference between the two processes.

\section{Other Testable Equivalences}

Any equivalence notion that coincides with a recursive replication-free test grammar is compatible with the RL algorithm described earlier. Several known equivalence notions fall in this category. Due to space limitations, we only present the test grammar that we propose for each of these notions, namely, Ready, Failure [10], Barb Acceptance, and Barb Failure [14] equivalences.

\begin{tabular}{|c|c|}
\hline Equivalence & Test Grammar \\
\hline Trace & $\mathcal{T}_{\text {1moment }}::=\omega \mid$ a.t \\
Ready & $\mathcal{T}_{\text {ready }}::=\omega \mid$ a.t $\mid\left\{a_{1}, \ldots, a_{n}\right\}$ \\
Failure & $\mathcal{T}_{\text {failure }}::=\omega \mid$ a.t $\mid\left\{\neg a_{1}, \ldots, \neg a_{n}\right\}$ \\
Barb Acceptance & $\mathcal{T}_{\text {BarbAcc }}::=\omega \mid$ a.t $\mid\left\{a_{1}, \ldots, a_{n}\right\}$ a.t \\
Barb Failure & $\mathcal{T}_{\text {BarbRef }}:=\omega \mid$ a.t $\mid\left\{\neg a_{1}, \ldots, \neg a_{n}\right\}$ a.t \\
\hline
\end{tabular}

A test of the form $\left\{a_{1}, \ldots, a_{n}\right\}$ consists in executing the actions $a_{1}, \ldots, a_{n}$ on $n$ copies of the current process, whereas the test of the form $\left\{a_{1}, \ldots, a_{n}\right\}$ a.t consists in executing actions $a_{1}, \ldots, a_{n}$ on respective copies of the current process, then executing action $a$ on another copy and if the latter succeeds proceeding with $t$.

\section{Conclusion}

The main contributions of this paper are (1) a completely new approach to estimate how far apart two LMPs are and (2) a new family of equivalences $(K$-moment) that are a good compromise between trace-equivalence and bisimulation. Indeed, we introduce a notion of divergence $\operatorname{div}_{K \text {-moment }}(. \|$. ) that can be estimated via some Monte-Carlo estimation using Reinforcement Learning algorithms. Traditional approaches, on the other hand, are based on costly complete 
calculations on the models. The RL approach therefore opens a way for analyzing huge systems and even infinite ones. Moreover, it can be adapted to other equivalences that can be tested via recursive replication-free test grammars.

For future work, we want to modify the construction of $\mathcal{M}_{K}$, in order to speed up the calculation, especially for situations like Ready equivalence, where the action set of the MDP is exponentially larger that the one of the LMP. Finally, since the LMP formalism is mathematically quite similar to the MDP, POMDP, and HMM formalisms, the next step will be to apply our approach on these formalisms. In particular, we could contribute to a theory of approximations.

\section{References}

1. R. Blute, J. Desharnais, A. Edalat, and P. Panangaden. Bisimulation for labelled Markov processes. In Proc. of the Twelfth IEEE Symposium On Logic In Computer Science, Warsaw, Poland, 1997.

2. F. Breugel, S. Shalit, and J. Worrell. Testing labelled Markov processes. In ICALP, volume 2380 of Lecture Notes in Computer Science, pages 537-548. Springer, 2002.

3. F. Van Breugel and J. Worrell. Approximating and computing behavioural distances in probabilistic transition systems. Theoretical Computer Science, 2006.

4. T. M. Cover and J. A. Thomas. Elements of Information Theory. Wiley, 1991.

5. J. Desharnais, F. Laviolette, K. Darsini Moturu, and S. Zhioua. Trace equivalence characterization through reinforcement learning. 2006. Accepted for publication in the 19th Canadian Conference on Artificial Intelligence.

6. E. Even-Dar and Y. Mansour. Learning rates for Q-learning. In COLT '01/EuroCOLT '01: Proc. of the 14th Annual Conference on Computational Learning Theory, pages 589-604, London, UK, 2001. Springer-Verlag.

7. C. N. Fiechter. Design and Analysis of Efficient Reinforcement Learning Algorithms. PhD thesis, Univ. of Pittsburgh, 1997.

8. A. Giacalone, C. Jou, and S. Smolka. Algebraic reasoning for probabilistic concurrent systems. In Proceedings of the Working Conference on Programming Concepts and Methods, IFIP TC2, 1990.

9. R.J. Van Glabbeek. The linear time - branching time spectrum ii. In CONCUR '93: Proceedings of the 4th International Conference on Concurrency Theory, pages 66-81, London, UK, 1993. Springer-Verlag.

10. C.-C. Jou and S. A. Smolka. Equivalences, congruences, and complete axiomatizations for probabilistic processes. In J. Baeten and J. Klop, editors, CONCUR 90 1st Int. Conf. on Concurrency Theory, number 458 in LNCS. Springer-Verlag, 1990.

11. L. P. Kaelbling, M. L. Littman, and A. P. Moore. Reinforcement learning: A survey. Journal of Artificial Intelligence Research, 4:237-285, 1996.

12. M. Kearns and S. Singh. Finite-sample convergence rates for q-learning and indirect algorithms. In Proc. of the 1998 conference on Advances in neural information processing systems II, pages 996-1002, Cambridge, MA, USA, 1999. MIT Press.

13. K. G. Larsen and A. Skou. Bisimulation through probabilistic testing. Inf. Comput., 94(1):1-28, 1991.

14. G. Lowe. Representing Nondeterministic and Probabilistic Behaviour in Reactive Processes. Technical report, Progr. Res. Group, Oxford University, 1993.

15. R. S. Sutton and A. G. Barto. Introduction to Reinforcement Learning. MIT Press, Cambridge, MA, USA, 1998.

16. C. Watkins.Learning from Delayed Rewards. PhD thesis, Univ. of Cambridge, 1989.

17. C. Watkins and P. Dayan. Q-learning. Machine Learning, 8:279-292, 1992. 